\title{
MAXIMAL OPERATORS AND HILBERT TRANSFORMS ALONG FLAT CURVES NEAR $L^{1}$
}

\author{
NEAL BEZ \\ (Received 3 July 2008; accepted 10 November 2008) \\ Communicated by G. A. Willis
}

\begin{abstract}
For a class of convex curves in $\mathbb{R}^{d}$ we prove that the corresponding maximal operator and Hilbert transform are of weak type $L \log L$. The point of interest here is that this class admits curves which are infinitely flat at the origin. We also prove an analogous weak type result for a class of nonconvex hypersurfaces.
\end{abstract}

2000 Mathematics subject classification: primary 42B20; secondary 42B25.

Keywords and phrases: maximal operator, Hilbert transform, weak type estimate.

\section{Introduction}

Given natural numbers $m$ and $d$ with $d \geq m+1$, let $\Gamma: \mathbb{R}^{m} \rightarrow \mathbb{R}^{d}$ be a smooth $m$ parameter surface in $\mathbb{R}^{d}$. Let the associated maximal operator, $M_{\Gamma}$, and Hilbert transform, $H_{\Gamma}$, be given by

$$
\begin{aligned}
& M_{\Gamma} f(x):=\sup _{h>0} \frac{1}{h^{m}}\left|\int_{|t| \in(0, h)} f(x-\Gamma(t)) d t\right|, \\
& H_{\Gamma} f(x):=\text { p.v. } \int_{\mathbb{R}^{m}} f(x-\Gamma(t)) K(t) d t,
\end{aligned}
$$

for appropriate functions $f$ on $\mathbb{R}^{d}$. Here, $K: \mathbb{R}^{m} \rightarrow \mathbb{R}$ is a Calderón-Zygmund kernel; that is, $K$ is $C^{\infty}$ away from the origin, homogeneous of degree $-d$ and $\int_{|t| \in[a, b]} K(t) d t=0$ for each $0<a<b$. In this paper we are concerned with the mapping properties of $M_{\Gamma}$ and $H_{\Gamma}$ on Orlicz spaces near $L^{1}$ and, in particular, with estimates of the form

$$
\left|\left\{x \in \mathbb{R}^{d}:|T f(x)|>\alpha\right\}\right| \leq \int_{\mathbb{R}^{d}} \Phi\left(\frac{C|f(x)|}{\alpha}\right) d x \quad \forall \alpha>0,
$$

The author acknowledges EPSRC support, including partial support from grant EP/E022340/1.

(C) 2009 Australian Mathematical Publishing Association, Inc. 1446-7887/2009 \$16.00 
where $\Phi:[0, \infty) \rightarrow[0, \infty)$ is a convex function and $T \in\left\{M_{\Gamma}, H_{\Gamma}\right\}$. As in [13], we shall say that $T$ is of weak type $\Phi(L)$ if (1.1) holds for all simple functions $f$ on $\mathbb{R}^{d}$.

Even for model cases of $\Gamma$ possessing nonvanishing Gaussian curvature, such as $m=1, d=2$ and $\Gamma(t)=\left(t, t^{2}\right)$, it is unknown whether $M_{\Gamma}$ and $H_{\Gamma}$ are of weak type $L$. Currently, the most progress on this question for the parabola is a weak type $L \log \log L$ estimate due to Seeger, Tao and Wright [13]; that is, with the usual abuse of notation, the estimate in (1.1) holds for both operators with $\Phi(t)=t \log \log (100+t)$. For closely related results, we note that both operators map the parabolic Hardy space to $L^{1, \infty}$ (Christ [7]) and map the smaller product-type Hardy space to the smaller Lorentz space $L^{1,2}$ (Seeger and Tao [12]). Christ [8] has also shown that both operators are not of weak type $L$ if $\Gamma$ is a 'piecewise linear parabola' (see also [3] for further results in this direction).

The main theorem in [13] which we state below covers a large class of hypersurfaces in $\mathbb{R}^{d}$ for $d \geq 2$, of which the parabolic plane curve is a special case.

THEOREM 1.1. [13] Let $\Sigma_{0}$ be a smooth compact hypersurface of $\mathbb{R}^{d}$ whose Gaussian curvature does not vanish to infinite order and let $v$ be a smooth and compactly supported density on $\Sigma_{0}$. Then the operator $f \mapsto \sup _{k \in \mathbb{Z}}\left|v_{k} * f\right|$ is of weak type $L \log \log L$, where

$$
\left\langle v_{k}, \psi\right\rangle:=\left\langle\nu, \psi\left(\delta_{2^{k}} \cdot\right)\right\rangle, \quad \delta_{t}:=\exp ((\log t) P),
$$

and $P$ is a real $d$ by $d$ matrix whose eigenvalues each have positive real part. Moreover, if the cancellation condition $\widehat{v}(0)=0$ holds then $f \mapsto \sum_{k \in \mathbb{Z}} v_{k} * f$ extends to an operator of weak type $L \log \log L$.

It is important for us to observe that weaker $L \log L$ estimates for the operators in Theorem 1.1 are true under weaker hypotheses. For the maximal operator, this is the case if $v$ is a compactly supported measure whose Fourier transform satisfies a decay estimate

$$
|\widehat{v}(\xi)| \leq C(1+|\xi|)^{-\tau} \quad \text { for some } \tau>0
$$

and, for the singular integral operator, if additionally we have the cancellation condition $\widehat{v}(0)=0$; see [13, Corollary 3.1]. The nonvanishing curvature hypothesis in Theorem 1.1 guarantees an estimate of the form (1.2) via van der Corput's lemma. This $L \log L$ result essentially reproved earlier work of Christ and Stein [9] who, for example, considered the class of homogeneous curves in $\mathbb{R}^{d}$ given by $\Gamma(t)=$ $\left(t^{\alpha_{1}}, \ldots, t^{\alpha_{d}}\right)$ for positive integers $\alpha_{j}$. We emphasize, however, that even for model cases when the codimension of $\Gamma$ is high, say $m=1, d=3$ and $\Gamma(t)=\left(t, t^{2}, t^{3}\right)$, there has been no improvement beyond $L \log L$.

Our first result in this note is an $L \log L$ estimate for a class of convex curves in $\mathbb{R}^{d}$ for $d \geq 2$ considered by Carbery et al. in [6] (see also [11] for very closely related work). Before describing this class, we highlight that certain curves which are infinitely flat at the origin are admitted into this class and therefore fall outside the scope of previous work. 
Suppose that $\Gamma(t)=\left(t, \gamma_{2}(t), \ldots, \gamma_{d}(t)\right)$, where each $\gamma_{j}$ belongs to $C^{d}(0, \infty)$. The next definition makes precise exactly what we mean by convex.

Definition 1.2. The curve $\left(t, \gamma_{2}(t), \ldots, \gamma_{d}(t)\right)$ is said to be convex if, for all $j=1, \ldots, d, D_{j}(t)>0$ for all $t>0$, where

$$
D_{j}(t):=\operatorname{det}\left(\begin{array}{cccc}
1 & \gamma_{2}^{\prime}(t) & \cdots & \gamma_{j}^{\prime}(t) \\
0 & \gamma_{2}^{\prime \prime}(t) & \cdots & \gamma_{j}^{\prime \prime}(t) \\
\vdots & \vdots & & \vdots \\
0 & \gamma_{2}^{(j)}(t) & \cdots & \gamma_{j}^{(j)}(t)
\end{array}\right) .
$$

Carbery et al. [6] showed that $M_{\Gamma}$ and $H_{\Gamma}$ are bounded on $L^{p}$ for all $p \in(1, \infty)$ if $\Gamma(t)=\left(t, \gamma_{2}(t), \ldots, \gamma_{d}(t)\right)$ is convex, odd, $\Gamma(0)=0$ and a certain curvature condition holds, namely,

$$
t h_{j}^{\prime}(t) \geq C_{d} h_{j}(t) \quad \forall t>0 \text { and } j=1, \ldots, d,
$$

where $h_{j}(t):=N_{j}(t) / D_{j-1}(t), D_{0}(t):=1$ and

$$
N_{j}(t):=\operatorname{det}\left(\begin{array}{cccc}
t & \gamma_{2}(t) & \cdots & \gamma_{j}(t) \\
1 & \gamma_{2}^{\prime}(t) & \cdots & \gamma_{j}^{\prime}(t) \\
\vdots & \vdots & & \vdots \\
0 & \gamma_{2}^{(j-1)}(t) & \cdots & \gamma_{j}^{(j-1)}(t)
\end{array}\right)
$$

This result is an extension of earlier work in [4] for plane curves. Our first main result is as follows.

THEOREM 1.3. Suppose that $\Gamma(t)=\left(t, \gamma_{2}(t), \ldots, \gamma_{d}(t)\right)$ is convex, odd, $\Gamma(0)=0$ and (1.3) holds. Then $M_{\Gamma}$ and $H_{\Gamma}$ are of weak type $L \log L$.

Our second result concerns the class of nonconvex plane curves and associated hypersurfaces considered recently in [1].

THEOREM 1.4. For $d \geq 2$, let $\Gamma: \mathbb{R}^{d-1} \rightarrow \mathbb{R}^{d}$ be a hypersurface of the form $\Gamma(y)=$ $(y, P(\gamma(|y|)))$ where $P$ is a real polynomial of degree no less than 2 and $\gamma$ satisfies the following conditions:

$$
\gamma \in C^{2}(0, \infty) \text {, convex on }[0, \infty), \gamma(0)=0 \text { and } \gamma^{\prime}(0) \geq 0 \text {. }
$$

If $\gamma$ is extended to be either even or odd, then the operators $M_{\Gamma}$ and $H_{\Gamma}$ are of weak type $L \log L$ if either (1) $d \geq 3$ or (2) $d=2$ and $P^{\prime}(0)=0$.

REMARKS. (1) In [1] it was shown that if $d=2$ and $P^{\prime}(0)$ is nonzero then $M_{\Gamma}$ and $H_{\Gamma}$ are bounded on $L^{p}$ for all $p \in(1, \infty)$ under the additional hypothesis that $\gamma^{\prime}$ is doubling; that is, there exists a constant $D \in(1, \infty)$ such that $\gamma^{\prime}(D t) \geq 2 \gamma^{\prime}(t)$ for 
each $t>0$. See [3] for certain negative results near $L^{1}$ when $P(s)=s$ and for a certain class of $\gamma$ for which $\gamma^{\prime}$ is doubling.

(2) The reason for the more relaxed hypothesis when $d \geq 3$ is that the curvature of $\mathbb{S}^{d-2}$ plays a significant role in the proof.

Notation. For nonnegative numbers $A$ and $B$, we write $A \lesssim B$ and $B \gtrsim A$ if $A \leq C B$, where the constant $C$ depends on at most $d$ and $\Gamma$. If $A \lesssim B \lesssim A$ then we may write $A \sim B$.

For $y \in \mathbb{R}^{d}$ and $r>0$, let $B(y, r)$ denote the open Euclidean ball with centre $y$ and radius $r$ given by

$$
B(y, r):=\left\{x \in \mathbb{R}^{d}:|x-y|<r\right\} .
$$

\section{Proof of Theorem 1.3}

We begin with the observation that if

$$
\mathcal{M}_{\Gamma} f(x):=\sup _{k \in \mathbb{Z}} \frac{1}{\lambda^{k}}\left|\int_{\lambda^{k}}^{\lambda^{k+1}} f(x-\Gamma(t)) d t\right|
$$

for some fixed $\lambda \in(1, \infty)$ depending on $d$ and $\Gamma$ to be determined later in the proof, then $M_{\Gamma} f \sim \mathcal{M}_{\Gamma} f$ for nonnegative functions $f$; thus, for the maximal operator, it suffices to consider $\mathcal{M}_{\Gamma}$. The main idea is to follow the arguments in [13] which led to the weaker $L \log L$ version of Theorem 1.1 under the weaker Fourier transform decay hypothesis, as described in the Introduction here. Further key ingredients to the proof are an appropriate Calderón-Zygmund theory and suitable control on the dilations in hand. In our flat setting determined by the curvature condition (1.3), we rely on [4] and [6] to provide these ingredients.

2.1. Dilations and decay estimates All of the work on the choice of dilations and proving the decay estimates that follow was done in [6] and thus we simply state their results; for motivation and proofs we refer the reader to [6]. The dilation matrices $\{A(t) \mid t>0\}$ are given by

$$
A(t):=\left(\begin{array}{cccc}
t & R_{1} t & \cdots & R_{d-1} R_{d-2} \cdots R_{1} t \\
\gamma_{2}(t) & R_{1} \gamma_{2}(t) & \cdots & R_{d-1} R_{d-2} \cdots R_{1} \gamma_{2}(t) \\
\vdots & \vdots & & \vdots \\
\gamma_{d}(t) & R_{1} \gamma_{d}(t) & \cdots & R_{d-1} R_{d-2} \cdots R_{1} \gamma_{d}(t)
\end{array}\right)
$$

where the differential operators $R_{1}, \ldots, R_{d-1}$ are given by

$$
R_{j} f:=\left(\frac{f}{h_{j}}\right)^{\prime} \frac{h_{j}^{2}}{h_{j}^{\prime}} \quad \text { for } j=1, \ldots, d-1 .
$$

We remark that the convexity hypothesis on $\Gamma$ ensures that $A(t)$ is well defined because all $h_{j}$ and $h_{j}^{\prime}$ are positive; see [11]. By [6, Lemma 5.3], $A(t)$ is lower 
triangular with $A(t)_{1,1}=t$ and $A(t)_{j, j}=h_{j}(t)$ for $j=2, \ldots, d$; hence $A(t)$ is invertible. Moreover, the curvature hypothesis (1.3) implies that the following Rivière condition holds (in fact, the full strength of the curvature hypothesis (1.3) is not needed to prove Proposition 2.1, and the assumption that each $h_{j}$ is doubling suffices; see [6]).

PROposition 2.1 [6]. There exist positive constants $C, \varepsilon$ depending on $\Gamma$ and $d$ such that $\left\|A(s)^{-1} A(t)\right\| \leq C(t / s)^{\varepsilon}$ for all $s \geq t>0$.

For each $k \in \mathbb{Z}$, we now define the normalized curves $\Gamma_{k}$ by

$$
\Gamma_{k}(t):=A\left(\lambda^{k}\right)^{-1} \Gamma\left(\lambda^{k} t\right) \quad \text { for each }|t| \in[1, \lambda] .
$$

Also, let the measures $\mu^{(k)}, \mu_{k}^{(k)}, H^{(k)}$ and $H_{k}^{(k)}$ be given by

$$
\begin{gathered}
\left\langle\mu^{(k)}, \psi\right\rangle:=\int_{1}^{\lambda} \psi\left(\Gamma_{k}(t)\right) d t, \quad\left\langle\mu_{k}^{(k)}, \psi\right\rangle:=\left\langle\mu^{(k)}, \psi\left(A\left(\lambda^{k}\right) \cdot\right)\right\rangle, \\
\left\langle H^{(k)}, \psi\right\rangle:=\int_{|t| \in[1, \lambda]} \psi\left(\Gamma_{k}(t)\right) \frac{d t}{t}, \quad\left\langle H_{k}^{(k)}, \psi\right\rangle:=\left\langle H^{(k)}, \psi\left(A\left(\lambda^{k}\right) \cdot\right)\right\rangle,
\end{gathered}
$$

so that $\mathcal{M}_{\Gamma} f=\sup _{k \in \mathbb{Z}}\left|\mu_{k}^{(k)} * f\right|$ and $H_{\Gamma} f=\sum_{k \in \mathbb{Z}} H_{k}^{(k)} * f$. The notation $\mu_{k}^{(k)}$ and $H_{k}^{(k)}$ may seem heavy-handed at first. The intention is to maintain the notation from [13] in the sense that $\mu_{k}^{(k)}$ and $H_{k}^{(k)}$ are $A\left(\lambda^{k}\right)$-dilates of the measures $\mu^{(k)}$ and $H^{(k)}$. In general, $\mu^{(k)}$ and $H^{(k)}$ will not be fixed as $k$ varies yet have the property that their Fourier transforms satisfy decay estimates independent of $k$, and in this sense are 'essentially' fixed. These crucial decay estimates are the content of the subsequent lemma. This was proved in [6, Section 5] via a variant of van der Corput's lemma (see [6, Proposition 3.1] for this variant).

Lemma 2.2 [6]. There exists $\tau \in(0,1)$ depending on $d$ such that

$$
\left|\widehat{\mu^{(k)}}(\xi)\right|+\left|\widehat{H^{(k)}}(\xi)\right| \lesssim(1+|\xi|)^{-\tau}
$$

for all $k \in \mathbb{Z}$ and all $\xi \in \mathbb{R}^{d}$.

2.2. Calderón-Zygmund theory In order to use the Calderón-Zygmund theory developed in [4], we shall define balls $\left\{B_{k}\right\}_{k \in \mathbb{Z}}$ satisfying the following conditions:

(B1) $\bigcup_{k \in \mathbb{Z}} B_{k}=\mathbb{R}^{d}$;

(B2) $\bigcap_{k \in \mathbb{Z}} B_{k}=\{0\}$;

(B3) each $B_{k}$ is open, balanced, convex and bounded;

(B4) $B_{k} \subset B_{k+1}$ for each $k \in \mathbb{Z}$;

(B5) for each $k,\left|B_{k+1}\right| \sim\left|B_{k}\right|$.

It follows from Proposition 2.1 that $\left\|A\left(\lambda^{k+1}\right)^{-1} A\left(\lambda^{k}\right)\right\|<1$ uniformly in $k$ if we choose $\lambda:=4^{\left\lceil 1+\left(\log _{2} C\right) /(2 \varepsilon)\right\rceil}$, where $C$ and $\varepsilon$ are those constants appearing in 
Proposition 2.1. Let $\widetilde{B_{k}}:=A\left(\lambda^{k}\right) B(0,1)$; one can check that (B1)-(B4) hold for the $\widetilde{B_{k}}$. We then define the collection of $B_{k}$ by

$$
\left\{B_{k}\right\}_{k \in \mathbb{Z}}:=\bigcup_{k \in \mathbb{Z}} \bigcup_{l=1}^{n(k)-1}\left\{E_{k}^{l}\right\}
$$

where, for each $k \in \mathbb{Z},\left\{E_{k}^{1}, \ldots, E_{k}^{n(k)}\right\}$ is a finite set of open, balanced, convex and bounded sets such that

$$
\widetilde{B_{k}}=E_{k}^{1} \subset E_{k}^{2} \subset \cdots \subset E_{k}^{n(k)}=\widetilde{B_{k+1}}
$$

and $\left|E_{k}^{l}\right| \leq 2\left|E_{k}^{l-1}\right|$ for $l=1, \ldots, n(k)-1$.

For each $k \in \mathbb{Z}$, observe that property (B3) allows us to define a norm $\|\cdot\|_{k}$ such that $B_{k}=\left\{x \in \mathbb{R}^{d}:\|x\|_{k}<1\right\}$.

Notation. For each $y \in \mathbb{R}^{d}, k \in \mathbb{Z}$ and $r>0$, let $B(y, k, r)$ be the open ball with centre $y$ and radius $r$ with respect to $\|\cdot\|_{k}$ given by

$$
B(y, k, r):=\left\{x \in \mathbb{R}^{d}:\|x-y\|_{k}<r\right\} .
$$

For each $k \in \mathbb{Z}, x \in \mathbb{R}^{d}$ and nonempty subset $S$ of $\mathbb{R}^{d}$, define

$$
\operatorname{dist}_{k}(x, S):=\inf \left\{\|x-s\|_{k}: s \in S\right\}
$$

We now state the Whitney-type decomposition relative to the balls in (2.2).

Proposition 2.3 [4]. There exists a constant A depending on $\Gamma$ and $d$ such that the following conditions hold.

(1) If $\Omega$ is any nonempty proper open subset of $\mathbb{R}^{d}$, then $\Omega=\bigcup_{B \in \mathfrak{W}} B$, where

$$
\mathfrak{W}:=\left\{B(x, k, 1) \mid x \in \Omega, k \in \mathbb{Z}, 5<\operatorname{dist}_{k}(x, \partial \Omega)<A\right\} .
$$

(2) If in addition $|\Omega|$ is finite, then we can find a sequence of disjoint balls $Q_{i}:=$ $B\left(x_{i}, k_{i}, 1\right) \in \mathfrak{W}$ such that $\Omega=\bigcup_{i} B\left(x_{i}, k_{i}, 3\right)$.

If $\mathfrak{B}:=\left\{B(y, k, A) \mid y \in \mathbb{R}^{d}, k \in \mathbb{Z}\right\}$, define the associated Hardy-Littlewood-type maximal function $M_{H L}$ by

$$
M_{H L} f(x):=\sup _{\substack{B \in \mathfrak{B} \\ x \in B}} \frac{1}{|B|} \int_{B}|f(y)| d y,
$$

where $A>0$ appears in Proposition 2.3. 
2.3. Conclusion of the proof of Theorem 1.3 Fix $\alpha>0$ and set

$$
\Omega:=\left\{x \in \mathbb{R}^{d} \mid M_{H L} f(x)>\alpha\right\} .
$$

By [4, Proposition 2.2], $M_{H L}$ is of weak type $L$ and therefore $|\Omega| \lesssim\|f\|_{1} / \alpha$. Next, apply Proposition 2.3 to obtain sequences $\left\{x_{i}\right\} \subseteq \Omega,\left\{j_{i}\right\} \subseteq \mathbb{Z}$ and disjoint Whitney cubes $\left\{Q_{i}\right\}$ such that:

(W1) $\Omega=\bigcup_{i} Q_{i}$;

(W2) $B\left(x_{i}, j_{i}, 1\right) \subseteq Q_{i} \subseteq B\left(x_{i}, j_{i}, 3\right)$;

(W3) $5<\inf \left\{\left\|x_{i}-y\right\|_{j_{i}}: y \in \partial \Omega\right\}<A$.

Also define $\Omega^{*}:=\bigcup_{i} B\left(x_{i}, j_{i}, C+10\right)$, where $C$ is the constant appearing in the statement of Proposition 2.1. Observe that (W1) implies that $\left|\Omega^{*}\right| \sim|\Omega|$; in particular, $\left|\Omega^{*}\right| \lesssim\|f\|_{1} / \alpha$.

By an analogue of the Lebesgue differentiation theorem, $|f(x)| \leq \alpha$ for $x \notin \Omega$. Our first decomposition is $f=g+\sum_{i} f_{Q_{i}}$, where

$$
f_{Q_{i}}(x):= \begin{cases}f(x) & \text { if } x \in Q_{i} \text { and }|f(x)|>\alpha, \\ 0 & \text { otherwise. }\end{cases}
$$

This decomposition is akin to that of classical Calderón-Zygmund theory. Next, decompose $f_{Q_{i}}$ further by letting

$$
f_{Q_{i}}^{n}(x):= \begin{cases}f_{Q_{i}}(x) & \text { if } \lambda^{(n-1) \tau} \alpha<\left|f_{Q_{i}}(x)\right| \leq \lambda^{n \tau} \alpha, \\ 0 & \text { otherwise, }\end{cases}
$$

where $\tau$ is the decay exponent from Lemma 2.2. Notice that $f_{Q_{i}}=\sum_{n \geq 1} f_{Q_{i}}^{n}$ and

$$
\sum_{n \geq 1} \frac{1}{\left|Q_{i}\right|} \int\left|f_{Q_{i}}^{n}(x)\right| d x \lesssim \alpha .
$$

Now let $g_{Q_{i}}^{n}:=\chi Q_{Q_{i}}\left|Q_{i}\right|^{-1} \int_{Q_{i}} f_{Q_{i}}^{n}$ and $b_{Q_{i}}^{n}:=f_{Q_{i}}^{n}-g_{Q_{i}}^{n}$. Furthermore, define $g^{n}:=$ $\sum_{i} g_{Q_{i}}^{n}, b^{n}:=\sum_{i} b_{Q_{i}}^{n}$ and $f^{n}:=\sum_{i} f_{Q_{i}}^{n}$. Observe that (2.3) implies that

$$
\sum_{n \geq 1}\left|g_{Q_{i}}^{n}(x)\right| \leq \chi Q_{i}(x) \sum_{n \geq 1} \frac{1}{\left|Q_{i}\right|} \int_{Q_{i}}\left|f_{Q_{i}}^{n}(y)\right| d y \lesssim \alpha \chi_{Q_{i}}(x) .
$$

Moreover, by (2.3) and (2.4),

$$
\sum_{n \geq 1}\left\|b_{Q_{i}}^{n}\right\|_{1} \lesssim \alpha\left|Q_{i}\right|
$$

We first consider $\mathcal{M}_{\Gamma}$, and the next step is to decompose the measures $\mu^{(k)}$, first by the following localization. Let $\phi \in \mathcal{S}\left(\mathbb{R}^{d}\right)$ have compact support in $B(0,1 / 2)$ with $\int \phi(x) d x=1$, and $\int x_{k} \phi(x) d x=0$ for all $k=1, \ldots, d$. Also define

$$
\mu^{(k), 0}:=\mu^{(k)} \quad \text { and } \quad \mu^{(k), n}:=\phi_{n} * \mu^{(k)},
$$


where $\phi_{n}(x):=\lambda^{n d} \phi\left(\lambda^{n} x\right)$ for each $n \geq 1$. For each $k \in \mathbb{Z}$ and $n \geq 1$ define the dilates

$$
\mu_{k}^{(k), n}:=\operatorname{det} A\left(\lambda^{k}\right)^{-1} \mu^{(k), n}\left(A\left(\lambda^{k}\right)^{-1} \cdot\right) .
$$

We are now in a position to decompose the operator $\mathcal{M}_{\Gamma}$ as

$$
\mathcal{M}_{\Gamma} f=\sup _{k \in \mathbb{Z}}\left|\mu_{k}^{(k)} * f\right| \leq \mathcal{M}_{I, 1}+\mathcal{M}_{I, 2}+\mathcal{M}_{I, 3}+\mathcal{M}_{I, 4}+\mathcal{M}_{I I},
$$

where

$$
\begin{aligned}
\mathcal{M}_{I, 1} & :=\sup _{k \in \mathbb{Z}}\left|\mu_{k}^{(k)} * g\right|, \\
\mathcal{M}_{I, 2} & :=\sup _{k \in \mathbb{Z}}\left|\mu_{k}^{(k)} * \sum_{n \geq 1} g^{n}\right|, \\
\mathcal{M}_{I, 3} & :=\sum_{n \geq 1} \sup _{k \in \mathbb{Z}}\left|\left(\mu_{k}^{(k)}-\mu_{k}^{(k), n}\right) * f^{n}\right|, \\
\mathcal{M}_{I, 4}: & =\sum_{m \geq 0} \sup _{k \in \mathbb{Z}}\left|\left(\mu_{k}^{(k), m+1}-\mu_{k}^{(k), m}\right) * \sum_{n>m} g^{n}\right|, \\
\mathcal{M}_{I I}: & =\sum_{n \geq 1} \sup _{k \in \mathbb{Z}}\left|\mu_{k}^{(k), n} * b^{n}\right| .
\end{aligned}
$$

In order to handle each $\mathcal{M}_{I, i}$ term we shall show that

$$
\sum_{i=1}^{4}\left\|\mathcal{M}_{I, i}\right\|_{2}^{2} \lesssim \alpha\|f\|_{1},
$$

Chebyshev's inequality immediately implying that the contribution from these terms is suitably under control. Before proving (2.6), we outline how we control the more difficult term $\mathcal{M}_{I I}$ using $L^{1}$ arguments. Recalling the definition of our balls $B_{j}$ from (2.1), for each $i$ let $l_{i}$ be the integer satisfying

$$
\widetilde{B_{l_{i}-1}} \subseteq B_{j_{i}} \subset \widetilde{B_{l_{i}}}
$$

For each $i$ and $n \geq 1$, set

$$
S_{n, i}:=\left\{k \in \mathbb{Z} \mid l_{i}-2 \leq k \leq l_{i}+\varepsilon^{-1} n\right\},
$$

where $\varepsilon$ appears in Proposition 2.1. Then $\mathcal{M}_{I I} \leq \mathcal{M}_{I I, 1}+\mathcal{M}_{I I, 2}$, where

$\mathcal{M}_{I I, 1}:=\sum_{n \geq 1} \sum_{i} \sum_{k \notin S_{n, i}}\left|\mu_{k}^{(k), n} * b_{Q_{i}}^{n}\right| \quad$ and $\quad \mathcal{M}_{I I, 2}:=\sum_{n \geq 1} \sum_{i} \sum_{k \in S_{n, i}}\left|\mu_{k}^{(k), n} * b_{Q_{i}}^{n}\right|$.

We claim that

$$
\left\|\mathcal{M}_{I I, 1}\right\|_{L^{1}\left(\mathbb{R}^{d} \backslash \Omega^{*}\right)} \lesssim\|f\|_{1}
$$


which, granted, implies that the contribution from $\mathcal{M}_{I I, 1}$ is under control by Chebyshev's inequality. For $\mathcal{M}_{I I, 2}$, notice that $\left\|\mu_{k}^{(k), n}\right\|_{1} \lesssim 1$ because of $\left\|\mu^{(k)}\right\| \lesssim 1$ and $L^{1}$ dilation invariance. Thus, by Chebyshev's inequality and the fact that $\left|S_{n, i}\right| \lesssim n$ for each $i$,

$$
\begin{aligned}
\left|\left\{x \in \mathbb{R}^{d} \mid \mathcal{M}_{I I, 2}(x)>\alpha / 6\right\}\right| & \lesssim \frac{1}{\alpha} \sum_{n \geq 1} \sum_{i} n\left\|b_{Q_{i}}^{n}\right\|_{1} \\
& \lesssim \int \frac{|f(x)|}{\alpha} \log \left(\frac{|f(x)|}{\alpha}+100\right) d x .
\end{aligned}
$$

The rest of the proof of Theorem 1.3 is then dedicated to (2.6) and (2.7).

From [6] we know that $\mathcal{M}_{\Gamma}$ is a bounded operator on $L^{2}$ and therefore

$$
\left\|\mathcal{M}_{I, 1}\right\|_{2}^{2} \lesssim\|g\|_{2}^{2} \lesssim \alpha\|f\|_{1} .
$$

Moreover, by (2.4),

$$
\left\|\sum_{n \geq 1} g^{n}\right\|_{2}^{2} \lesssim \alpha \sum_{n \geq 1} \sum_{i} \int\left|g_{Q_{i}}^{n}(x)\right| d x=\alpha \sum_{n \geq 1} \sum_{i} \int\left|f_{Q_{i}}^{n}(x)\right| d x \leq \alpha\|f\|_{1} .
$$

Thus, $\left\|\mathcal{M}_{I, 2}\right\|_{2}^{2} \lesssim \alpha\|f\|_{1}$. To handle $\mathcal{M}_{I, 3}$ and $\mathcal{M}_{I, 4}$ we use the following estimates concerning our localized measures.

LEMMA 2.4. For each $m \geq 0$,

$$
\left\|\sup _{k \in \mathbb{Z}}\left|\left(\mu_{k}^{(k), m+1}-\mu_{k}^{(k), m}\right) * f\right|\right\|_{2} \lesssim \lambda^{-m \tau}\|f\|_{2} .
$$

PROOF. By dominating the supremum over $k \in \mathbb{Z}$ with an $l^{2}(\mathbb{Z})$-sum and Plancherel's theorem, it suffices to show that

$$
\sum_{k \in \mathbb{Z}}\left|\mu^{\widehat{(k), m+1}}\left(A\left(\lambda^{k}\right)^{*} \xi\right)-\widehat{\mu^{(k), m}}\left(A\left(\lambda^{k}\right)^{*} \xi\right)\right|^{2} \lesssim \lambda^{-2 m \tau}
$$

for each $\xi \neq 0$. We claim that

$$
\left|\widehat{\mu^{(k), m+1}}(\xi)-\widehat{\mu^{(k), m}}(\xi)\right| \lesssim \lambda^{-m \tau} \min \left(\lambda^{-m}|\xi|,\left(\lambda^{-m}|\xi|\right)^{-1}\right) .
$$

That (2.9) implies (2.8) easily follows because Proposition 2.1 allows us to estimate the left-hand side of (2.8) by a convergent geometric series. Estimate (2.9) follows from the uniform decay estimate on the Fourier transform of $\mu^{(k)}$ using Lemma 2.2 and properties of $\phi$.

Lemma 2.4 implies that

$$
\begin{aligned}
\left\|\mathcal{M}_{I, 3}\right\|_{2} & \leq \sum_{n \geq 1} \sum_{m \geq n}\left\|\sup _{k \in \mathbb{Z}}\left|\left(\mu_{k}^{(k), m+1}-\mu_{k}^{(k), m}\right) * f^{n}\right|\right\|_{2} \lesssim \sum_{n \geq 1} \lambda^{-n \tau}\left\|f^{n}\right\|_{2} \\
& \lesssim \alpha^{1 / 2}\|f\|_{1}^{1 / 2} \sum_{n \geq 1} \lambda^{-n \tau / 2} \lesssim \alpha^{1 / 2}\|f\|_{1}^{1 / 2}
\end{aligned}
$$


and, moreover,

$$
\left\|\mathcal{M}_{I, 4}\right\|_{2} \lesssim \sum_{m \geq 0} \lambda^{-m \tau}\left\|\sum_{n>m} g^{n}\right\|_{2} \lesssim \alpha\|f\|_{1}
$$

This concludes the proof of (2.6).

To see (2.7), we first claim that for fixed $i$, those $k$ such that $k \leq l_{i}-2$ do not contribute to $\left\|\mathcal{M}_{I I, 1}\right\|_{L^{1}\left(\mathbb{R}^{d} \backslash \Omega^{*}\right)}$. To see this, write

$$
A\left(\lambda^{k}\right) \operatorname{supp} \mu^{(k)}=\left\{A\left(\lambda^{k+1}\right) \Gamma_{k+1}\left(\lambda^{-1} t\right) \mid t \in[1, \lambda]\right\} .
$$

By Proposition 2.1 and the fact that $\Gamma_{k+1}(s)=A\left(\lambda^{k+1}\right)^{-1} A\left(\lambda^{k+1} s\right) e_{1}$, where $e_{1}$ is the first canonical basis vector of $\mathbb{R}^{d}$, it follows that

$$
A\left(\lambda^{k}\right) \operatorname{supp} \mu^{(k)} \subseteq C \widetilde{B_{k+1}} \subseteq C \widetilde{B_{l_{i}-1}} \subseteq C B_{j_{i}} .
$$

Here, $C$ is the constant appearing in Proposition 2.1. Since each $\phi_{n}$ is supported in $B(0,1 / 2)$, it follows that $A\left(\lambda^{k}\right) \operatorname{supp} \phi_{n} \subseteq B_{j_{i}}$ and hence, $\operatorname{supp}\left(\mu_{k}^{(k), n} * b_{Q_{i}}^{n}\right) \subseteq \Omega^{*}$. So it suffices to prove that

$$
\sum_{i} \sum_{n \geq 1} \sum_{k \geq l_{i}+\varepsilon^{-1} n}\left\|\mu_{k}^{(k), n} * b_{Q_{i}}^{n}\right\|_{1} \lesssim\|f\|_{1} .
$$

For fixed $x \in \mathbb{R}^{d}$ we use the cancellation in $b_{Q_{i}}^{n}$ and Taylor's theorem to get

$$
\begin{aligned}
\operatorname{det} & A\left(\lambda^{k}\right)\left(\mu_{k}^{(k), n} * b_{Q_{i}}^{n}(x)\right) \\
= & \int_{Q_{i}} b_{Q_{i}}^{n}(y)\left[\mu^{(k), n}\left(A\left(\lambda^{k}\right)^{-1}(x-y)\right)-\mu^{(k), n}\left(A\left(\lambda^{k}\right)\left(x-x_{i}\right)\right)\right] d y \\
= & \int_{0}^{1} \int_{Q_{i}} b_{Q_{i}}^{n}(y)\left\langle A\left(\lambda^{k}\right)^{-1}\left(x_{i}-y\right), \nabla \mu^{(k), n}\left(A\left(\lambda^{k}\right)^{-1}\left(x-x_{i}+t\left(x_{i}-y\right)\right)\right)\right\rangle d y d t .
\end{aligned}
$$

For $y \in Q_{i}$,

$$
A\left(\lambda^{k}\right)^{-1}\left(x_{i}-y\right) \in A\left(\lambda^{k}\right)^{-1} A\left(\lambda^{l_{i}}\right) B(0,3) .
$$

Since $k \geq l_{i}$, it follows by Proposition 2.1 that $\left|A\left(\lambda^{k}\right)^{-1}\left(x_{i}-y\right)\right| \lesssim \lambda^{\left(l_{i}-k\right) \varepsilon}$. Also, $\left\|\nabla \mu^{(k), n}\right\|_{1} \lesssim \lambda^{n}$ because $\left\|\mu^{(k)}\right\| \lesssim 1$. Therefore,

$$
\int\left|\mu_{k}^{(k), n} * b_{Q_{i}}^{n}(x)\right| d x \lesssim \lambda^{\left(l_{i}-k\right) \varepsilon+n}\left\|b_{Q_{i}}^{n}\right\|_{1},
$$

and by (2.5) this implies that

$$
\sum_{i} \sum_{n \geq 1} \sum_{k \geq l_{i}+\varepsilon^{-1} n}\left\|\mu_{k}^{(k), n} * b_{Q_{i}}^{n}\right\|_{1} \lesssim \sum_{i} \sum_{n \geq 1}\left\|b_{Q_{i}}^{n}\right\|_{1} \lesssim \alpha|\Omega| \lesssim\|f\|_{1}
$$

as required. This concludes the proof of Theorem 1.3 for $M_{\Gamma}$. As in [13], a very similar argument works for $H_{\Gamma}$. Indeed, no further ideas beyond those in [13] and those contained in our proof of Theorem 1.3 for the maximal operator are needed. Thus we refer the reader to [13] (or to [2]) for a fleshed-out proof for $H_{\Gamma}$. 


\section{Proof of Theorem 1.4}

Without loss of generality $P(0)=0$, so $P(s)=\sum_{j=1}^{n} p_{j} s^{j}$ for some real numbers $p_{j}$. As in [1], we use a decomposition of the form

$$
(0, \infty)=\left.\left.\bigcup_{j \in \mathfrak{J}} \gamma\right|_{(0, \infty)} ^{-1}\left(G_{j}\right) \cup \bigcup_{l \in \mathfrak{L}} \gamma\right|_{(0, \infty)} ^{-1}\left(D_{l}\right),
$$

which is based on properties of $P$. Here, $\mathfrak{J}$ are those $j=1, \ldots, n$ such that the interval $G_{j}:=\left(\kappa\left|z_{j}\right|, \kappa^{-1}\left|z_{j+1}\right|\right)$ is nonempty, where $\kappa$ is some constant depending only on $n$ and the $z_{j}$ are the roots of $P$ ordered as

$$
0=\left|z_{1}\right| \leq\left|z_{2}\right| \leq \cdots \leq\left|z_{n}\right|
$$

with $z_{n+1}:=\infty$. We may choose the $D_{l}$ to be disjoint and such that if $D_{l}=\left(\alpha_{l}, \beta_{l}\right)$ then $\alpha_{l} \sim \beta_{l}$. Moreover, $\gamma^{-1}\left(\alpha_{l}\right) \sim \gamma^{-1}\left(\beta_{l}\right)$ since $\gamma$ is convex and passes through the origin (see, for example, [1]). Therefore, if

$$
\mathcal{M}_{\Gamma, I} f(x):=\sup _{k \in \mathbb{Z}} 2^{-(d-1) k}\left|\int_{\left.|t| \in\left[2^{k}, 2^{k+1}\right] \cap \gamma\right|_{(0, \infty)} ^{-1}(I)} f(x-\Gamma(t)) d t\right| \quad \text { for } I \subset(0, \infty)
$$

then each $\mathcal{M}_{\Gamma, D_{l}}$ is bounded on $L^{1}$ by Minkowski's inequality. Since the cardinalities of $\mathfrak{J}$ and $\mathfrak{L}$ cannot exceed $n$, we only need consider each $\mathcal{M}_{\Gamma, G_{j}}$. The point is that $P$ is suitably under control on each $G_{j}$ in the following sense.

LEMma 3.1. There exists a constant $C_{n}>1$ such that, for any $\kappa \geq C_{n}$ :

(1) $|P(s)| \sim\left|p_{j}\right| s^{j}$ for all $j \in \mathfrak{J}$ and $s \in G_{j}$;

(2) $P^{\prime}(s) / P(s)>0$ for all $j \in \mathfrak{J}$ and $s \in G_{j}$;

(3) $s P^{\prime}(s) / P(s) \sim 1$ for all $j \in \mathfrak{J}$ and $s \in G_{j}$;

(4) $P^{\prime \prime}(s) / P(s)>0$ and $s^{2} P^{\prime \prime}(s) / P(s) \sim 1$ for all $j \in \mathfrak{J} \backslash\{1\}$ and $s \in G_{j}$.

Lemma 3.1 appeared in this form in [2] but the ideas originated in [5]. We fix $j \in \mathfrak{J}$ and proceed as in our proof of Theorem 1.3. The appropriate $d$ by $d$ dilation matrices $\{A(t) \mid t>0\}$ are given by

$$
A(t)_{k, l}:= \begin{cases}t & \text { for } k=l \text { and } k=1, \ldots, d-1 \\ \left|p_{j}\right| \gamma(t)^{j} & \text { for } k=l=d \\ 0 & \text { for } k \neq l .\end{cases}
$$

The convexity of $\gamma$ implies that the Rivière condition in Proposition 2.1 holds. We again use the Calderón-Zygmund theory developed in [4] with $\widetilde{B_{k}}:=A\left(2^{k}\right) B(0,1)$ and an appropriately chosen supersequence of $B_{k}$ to ensure that condition (B5) holds. In fact, our only job is to verify that an appropriate decay estimate holds. We normalize $\Gamma$ in the analogous way; that is, for each $k \in \mathbb{Z}, \Gamma_{k}$ is given by

$$
\Gamma_{k}(t):=A\left(2^{k}\right)^{-1} \Gamma\left(2^{k} t\right) \quad \text { for } t \in \mathbb{R}^{d-1} .
$$


Therefore, $\Gamma_{k}(t)=\left(t, \gamma_{k}(|t|)\right)$ where

$$
\gamma_{k}:=\frac{P\left(\gamma\left(2^{k} \cdot\right)\right)}{\left|p_{j}\right| \gamma\left(2^{k}\right)^{j}}
$$

We write $\mathcal{M}_{\Gamma, G_{j}} f=\sup _{k \in \mathbb{Z}}\left|\mu_{k}^{(k)} * f\right|$ where

$$
\left\langle\mu^{(k)}, \psi\right\rangle:=\int_{|t| \in I_{k}} \psi\left(\Gamma_{k}(t)\right) d t, \quad\left\langle\mu_{k}^{(k)}, \psi\right\rangle:=\left\langle\mu^{(k)}, \psi\left(A\left(2^{k}\right) \cdot\right)\right\rangle
$$

and $I_{k}:=\left.[1,2] \cap 2^{-k} \gamma\right|_{(0, \infty)} ^{-1}\left(G_{j}\right)$.

LEMMA 3.2. For each $k \in \mathbb{Z}$ and each $\xi \in \mathbb{R}^{d}$,

$$
\left|\widehat{\mu^{(k)}}(\xi)\right| \lesssim(1+|\xi|)^{-1 / 2}
$$

PROOF. Under hypothesis (1) of Theorem 1.4 we capitalize on the decay of the Fourier transform of surface measure on $\mathbb{S}^{d-2}$. The details of this argument can be found in [1, Lemma 3.1] with the main impetus of ideas originating in [10]. Assuming that (2) of Theorem 1.4 holds, we claim that

$$
\left|\gamma_{k}^{\prime \prime}(t)\right| \gtrsim\left|\gamma_{k}^{\prime}(t)\right| \gtrsim\left|\gamma_{k}(t)\right| \gtrsim 1 \quad \forall t \in I_{k} .
$$

Given the claim, the proof of Lemma 3.2 follows from standard arguments using van der Corput's lemma.

To prove the claim, it is immediate that Lemma 3.1 and (1.4) give $\left|\gamma_{k}(t)\right| \gtrsim 1$ for all $t \in I_{k}$. Moreover, (1.4) in Theorem 1.4 implies that $t \gamma^{\prime}(t) \geq \gamma(t)$ for all $t>0$. Thus, by Lemma 3.1,

$$
\frac{\left|\gamma_{k}^{\prime}(t)\right|}{\left|\gamma_{k}(t)\right|}=2^{k} \frac{\left|P^{\prime}\left(\gamma\left(2^{k} t\right)\right)\right|}{\left|P\left(\gamma\left(2^{k} t\right)\right)\right|} \gamma^{\prime}\left(2^{k} t\right) \gtrsim 2^{k} \frac{\gamma^{\prime}\left(2^{k} t\right)}{\gamma\left(2^{k} t\right)} \gtrsim 1,
$$

which implies that $\left|\gamma_{k}^{\prime}(t)\right| \gtrsim 1$. For the remaining assertion, observe that

$$
\frac{P^{\prime \prime}\left(\gamma\left(2^{k} t\right)\right) \gamma^{\prime}\left(2^{k} t\right)}{P^{\prime}\left(\gamma\left(2^{k} t\right)\right)} \text { and } \frac{\gamma^{\prime \prime}\left(2^{k} t\right)}{\gamma^{\prime}\left(2^{k} t\right)}
$$

are both positive on $I_{k}$ by Lemma 3.1. Therefore,

$$
\frac{\left|\gamma_{k}^{\prime \prime}(t)\right|}{\left|\gamma_{k}^{\prime}(t)\right|}=2^{k}\left|\frac{P^{\prime \prime}\left(\gamma\left(2^{k} t\right)\right) \gamma^{\prime}\left(2^{k} t\right)}{P^{\prime}\left(\gamma\left(2^{k} t\right)\right)}+\frac{\gamma^{\prime \prime}\left(2^{k} t\right)}{\gamma^{\prime}\left(2^{k}\right)}\right| \geq 2^{k} \frac{P^{\prime \prime}\left(\gamma\left(2^{k} t\right)\right) \gamma^{\prime}\left(2^{k} t\right)}{P^{\prime}\left(\gamma\left(2^{k} t\right)\right)} \gtrsim 1,
$$

where the last bound follows from another application of Lemma 3.1. This completes the proof of Lemma 3.2.

One can easily verify that the corresponding decay estimates also hold for the normalized measures associated with $H_{\Gamma}$. Thus, Theorem 1.4 now follows from the argument used to prove Theorem 1.3.

REMARK. It follows from the proof of Theorem 1.4 that the conclusion holds with a finite constant $C$ which one can take to be independent of the coefficients of $P$. 


\section{Acknowledgements}

This work formed part of my $\mathrm{PhD}$ thesis at the University of Edinburgh, and I would like to thank Jim Wright for his discussions on this work and continuing guidance.

\section{References}

[1] N. Bez, ' $L^{p}$-boundedness for the Hilbert transform and maximal operator along a class of nonconvex curves', Proc. Amer. Math. Soc. 135 (2007), 151-161.

[2] - Nonisotropic Operators Arising in the Method of Rotations, PhD Thesis, University of Edinburgh (2007).

[3] _ - 'Maximal operators along piecewise linear curves near $L^{1}$ ', Indiana Univ. Math. J. 58 (2009), 1639-1658.

[4] A. Carbery, M. Christ, J. Vance, S. Wainger and D. Watson, 'Operators associated to flat plane curves: $L^{p}$ estimates via dilation methods', Duke Math. J. 59 (1989), 677-700.

[5] A. Carbery, F. Ricci and J. Wright, 'Maximal functions and Hilbert transforms associated to polynomials', Rev. Mat. Ibero. 14 (1998), 117-144.

[6] A. Carbery, J. Vance, S. Wainger and D. Watson, 'The Hilbert transform and maximal function along flat curves, dilations, and differential equations', Amer. J. Math. 116 (1994), 1203-1239.

[7] M. Christ, 'Weak type (1,1) bounds for rough operators', Ann. of Math. (2) 128 (1988), 19-42.

[8] _ 'Examples of singular maximal functions unbounded on $L^{p}$, Publ. Mat. 35 (1991), 269-279.

[9] M. Christ and E. M. Stein, 'A remark on singular Calderón-Zygmund theory', Proc. Amer. Math. Soc. 99 (1987), 71-75.

[10] W. Kim, S. Wainger, J. Wright and S. Ziesler, 'Singular integrals and maximal functions associated to surfaces of revolution', Bull. London Math. Soc. 28 (1996), 291-296.

[11] A. Nagel, J. Vance, S. Wainger and D. Weinberg, 'The Hilbert transform for convex curves in $\mathbb{R}^{n}$, Amer. J. Math. 108 (1986), 485-504.

[12] A. Seeger and T. Tao, 'Sharp Lorentz space estimates for rough operators', Math. Ann. 320 (2001), $381-415$.

[13] A. Seeger, T. Tao and J. Wright, 'Singular maximal functions and Radon transforms near $L^{1}$, Amer. J. Math. 126 (2004), 607-647.

NEAL BEZ, School of Mathematics, The Watson Building, University of Birmingham, Edgbaston, Birmingham B15 2TT, UK e-mail: bezn@maths.bham.ac.uk

Current address: Department of Mathematics, University Gardens, University of Glasgow, G12 8QW, UK

e-mail:n.bez@maths.gla.ac.uk 